\title{
Preliminary Validation of a Chinese Version of the Comprehensive Assessment of Acceptance and Commitment Therapy Processes
}

\section{Yue Chen}

Key Laboratory of Mental Health, Institute of Psychology,Chinese Academy of Sciences https://orcid.org/0000-0002-4173-2465

Houyuan Luo

private practice

\section{Shujuan Wang}

Key Laboratory of Mental Health,Institute of Psychology,Chinese Academy of sciences

\section{Xiaoyu Bai}

Key Laboratory of Mental Health,Institute of Psychology,Chinese Academy of Sciences

\section{Zhuohong Zhu ( $\nabla$ zhuzh@psych.ac.cn )}

Key Laboratory of Mental Health,Institute of Psychology,Chinese Academy of Sciences

https://orcid.org/0000-0002-3426-3402

\section{Research}

Keywords: Civil servants, psychological flexibility, CompACT, assessment

Posted Date: May 7th, 2020

DOl: https://doi.org/10.21203/rs.3.rs-26442/v1

License: (c) (i) This work is licensed under a Creative Commons Attribution 4.0 International License.

Read Full License

Version of Record: A version of this preprint was published at Current Psychology on February 19th, 2022. See the published version at https://doi.org/10.1007/s12144-021-02654-y. 


\title{
Preliminary Validation of a Chinese Version of the Comprehensive Assessment of Acceptance and Commitment Therapy Processes
}

\author{
Yue Chen \\ Institute of Psychology, Chinese Academy of Sciences, Beijing, China \\ Houyuan Luo \\ Toronto, Ontario, Canada \\ Shujuan Wang \\ Institute of Psychology, Chinese Academy of Sciences, Beijing, China \\ Xiaoyu Bai \\ Institute of Psychology, Chinese Academy of Sciences, Beijing, China \\ Zhuohong Zhu* \\ Institute of Psychology, Chinese Academy of Sciences, Beijing, China
}

Author Note

Yue Chen, Shujuan Wang, Xiaoyu Bai and Zhuohong Zhu , Key Laboratory of

Mental Health, Institute of Psychology, Chinese Academy of Sciences ; Houyuan Luo, Private practice, Toronto, Ontario, Canada;

Correspondence concerning this article should be addressed to Zhuohong Zhu, Key

Laboratory of Mental Health, Institute of Psychology, Chinese Academy of

Sciences, 16 Lincui Road, Chaoyang District, Beijing 100101, People's Republic of

China. Email: zhuzh@psych.ac.cn 


\begin{abstract}
Civil servants are bridges that connect the general public and the government in China. Because their mental health may influence the rise and fall of the country, it is important to study their mental health and living conditions. The Comprehensive Assessment of Acceptance and Commitment Therapy Processes (CompACT) is a general measure of ACT processes and reflects individual psychological flexibility. We investigated the psychometric properties of CompACT in a non-clinical sample of civil servants. The Chinese CompACT demonstrated acceptable internal consistency (Cronbach $\alpha=0.87)$ and reliability (Guttman split-half coefficient $=0.69)$. Confirmatory factor analysis provided support for the three-factor model. Our findings suggest that the Chinese version of the CompACT has acceptable psychometric properties and is a valid instrument for the assessment of psychological flexibility.
\end{abstract}

Keywords: Civil servants, psychological flexibility, CompACT, assessment 


\section{Introduction}

\section{Process-Based Therapy}

Steven Hayes argued that cognitive-behavioural therapy (CBT) could be roughly categorized into three historical waves. The first wave was behavior therapy, which was designed to change overt behavior based on stimulus-response learning theory. The second was the era of classic CBT, which focused on maladaptive thinking patterns in emotions and behaviors, and was in favor of computer metaphors (Hayes, 2004; Hayes \& Hofmann, 2017). These two generations of methods and concepts focused on alleviating symptoms of psychological disorders, which led to the development of evidence-based treatments. However, comorbidity and client heterogeneity were common within syndromal groups, which made it difficult to diagnosis of diseases through a single application of a CBT protocol to a single disorder. A new generation of evidence-based care called Process-based Therapies (PBT; Hofmann \& Hayes, 2019) emerged to study core mediators and moderators based on testable theories. Process-based interventions emphasize the process of change. They could alleviate diverse symptoms and promote well-being by influencing core psychological processes, which can have broad effects on well-being (Dindo, Van Liew, \& Arch, 2017; Hayes, Pistorello, \& Levin, 2012). The methods used by the current third wave of CBT are often more experiential and underlying philosophies are more contextualistic (Hayes, 2004). This set of approaches includes Acceptance and Commitment Therapy (ACT), Dialectical Behavior Therapy (DBT), 
Mindfulness-based Cognitive Therapy (MBCT), Functional Analytic Psychotherapy (FAP), and others. These approaches focus more on the relationship between individuals and their thoughts as well as emotions, instead of the content. As a result, they emphasize concepts such as mindfulness, emotions, acceptance, the relationship, values, goals, and meta-cognition (Hayes \& Hofmann, 2017).

\section{Acceptance and Commitment Therapy}

As a representative treatment of the third-wave CBT, Acceptance and Commitment Therapy (ACT) is a trans-diagnostic therapeutic approach that conceptualizes psychological suffering as primarily a function of attempts to avoid unwanted private experiences (experiential avoidance) as well as a resultant or contingent reduction in personally-meaningful pursuits (Francis, Dawson, \& GolijaniMoghaddam, 2016). Therefore, ACT encourages clients to change the relationship between their thoughts and physical sensations through mechanisms of acceptance, mindfulness, and value-based action (Hayes, 2004). The ultimate goal of ACT is not to change diverse symptoms or conditions, but to increase psychological flexibility, which is the ability to behave consistently with ones' values, even if their thoughts and feelings oppose taking valued action, while being mindful of experiences in the present moment in a nonjudgmental and accepting way (Livheim et al., 2015).

Psychological flexibility can be conceptualized as a product of six interrelated but overlapping sub-processes: acceptance, diffusion, self as context, present moment awareness, values, and committed action (Hayes, Luoma, Bond, Masuda, \& Lillis, 
2006). According to the hexaflex model, ACT is more focused on testing cognitive processes than other psychometric methods. Further, higher levels of psychological flexibility have been found to be associated with lower levels of psychological distress and improved quality of life (Hayes, Levin, Plumb-Vilardaga, Villatte, \& Pistorello, 2013). Psychological flexibility has been shown to be associated with effective treatment of trichotillomania (Lee et al., 2018), depression (Bai, Luo, Zhang, Wu, \& Chi, 2019), and trauma-related problems (McLean \& Follette, 2016).

Psychological flexibility can reflect the mental health of individuals by relating to whether or not they can effectively deal with their negative emotions and feelings.

Although the most commonly used measure of psychological flexibility is the Acceptance and Action Questionnaire (AAQ-II, Bond et al., 2011), it only focuses on the process of acceptance and diffusion. It has been proved to overlap with general distress measures (Wolgast, 2014). Other measures, such as the Brief Experiential Avoidance Questionnaire (Gámez et al., 2014), the Cognitive Fusion Questionnaire (CFQ, Gillanders et al., 2014), and the Valued Living Questionnaire (Wilson, Sandoz, Kitchens, \& Roberts, 2010), examine the single process of psychological flexibility. To address the gap, Francis et al. compiled the Comprehensive Assessment of Acceptance and Commitment Therapy Processes (CompACT) in 2015 (Francis et al., 2016) to develop a general measure of ACT processes. The CompACT is a 23-item scale that includes three factors: openness to experience, behavioral awareness, and valued action. The three-factor structure is concordant with recent accounts of psychological flexibility in terms of three dyadic processes (Hayes, Villatte, Levin, \& 
Hildebrandt, 2011). The CompACT has shown the potential to be a good general measure of ACT processes (Francis et al., 2016). However, a Chinese version of the CompACT is needed to measure psychological flexibility in a Chinese cultural context.

\section{Mental Health of Chinese Civil Servants}

In China, civil servants are a special group that acts as a bridge between the general public and the government (Zhu, Chen, Ou, Geng, \& Jiang, 2014) and plays a relatively important role in influencing the rise and fall of a country (Lu \& Liang, 2016). Civil servants work in the governmental bureaucracy, manage social and public affairs, exercise state power, and perform national administrations. At the same time, they are responsible for delivering public service, and state finance are responsible for providing wages and benefits ( $\mathrm{Li}, 2014)$. Thus, the efficiency of servants directly affects the level of efficiency of the administration and the impartiality of social management; it further affects society and economic development (Liu \& Liu, 2006). However, given the nature of their work in local governments, Chinese civil servants lack decision-making power, and their working arrangements lack flexibility (Jia et al., 2014).

Meanwhile, as society evolves and reforms deepen, the government's management of civil servants has become more standardized and stringent (Hao, Hong, Xu, Zhou, \& Xie, 2015a), which causes stresses to the group. Compared with people in other occupations, Chinese civil servants have been shown to be at a higher 
risk of suffering as a result of several psychosocial stressors, such as traditional bureaucratic culture, the heavy workload and responsibilities, infrastructural problems and conflicts, and intense competition (Chen, 2005; Nian, 2004; Y. Wang, 2008).

Burn-out and other negative moods also worsen the psychological health of Chinese civil servants (Hao et al., 2015a). Therefore, studying their mental health is essential.

\section{Aims and objectives}

Many questionnaires have been used to measure mental health symptoms of Chinese civil servants, such as the Hamilton Depression Rating Scale (HAMD; Huang, Cao, Liu, Yao, \& Rui, 2018), the twelve-item Short Form Health Survey (SF12; Lu \& Liang, 2016), the General Health Questionnaire (GHQ-12; Liang, Wang, \& Yin, 2016), the Symptom Checklist 90 (SCL-90, Zhu et al., 2014), and the Civil Servants Stress Scale (CSSS, Hao et al., 2015a). However, no studies have measured the psychological flexibility of them. The present study examined the psychometric properties and performance of a Chinese version of the CompACT (Ch-CompACT) for a sample of Chinese civil servants. The present study can be a comprehensive instrument to evaluate the psychological flexibility of Chinese adults. This study aimed to apply the Ch-CompACT to Chinese civil servants and a control group, test its psychometric properties (including factor analysis and reliability), and compare the instrument with measures of depression, anxiety, stress level, and life satisfaction. 


\section{Method}

\section{Procedure}

Preparation of the initial Chinese Version of the CompACT . First, we translated the items from the CompACT into Chinese and then back-translated them into English. The procedures were as follows: Two postgraduate students translated the English version into Chinese independently. Then experts in psychology and the ACT reviewed and revised it until they reached a consensus. Then, another postgraduate student translated it back to English and compared it with the original English version to ensure conceptual equivalence.

Revision of the initial Chinese Version of the CompACT . Considering the cultural differences, some items were deleted or modified to fit the Chinese context and facilitate participant understanding. Based on expert criteria, we removed item 1 ("I tell myself that I shouldn't have certain thoughts"), item 2 ("I try to stay busy to keep thoughts or feelings from coming"), Item 7 ("I can take thoughts and feelings as they come, without attempting to control or avoid them"), Item 8 ("I am willing to fully experience whatever thoughts, feelings and sensations come up for me, without trying to change or defend against them "), item 19 ("I can keep going with something when it's important to me"), and item 23 ("I can identify the things that really matter to me in life and pursue them"). These items may be hard to understand for Chinese people. Some items were selected from the AAQ- II, CFQ, and VQ and added to the scale. The items from AAQ-II included: "I worry about not being able to control my 
worries and feelings", "My painful experiences and memories make it difficult for me to live a life that I would value", and "I'm afraid of my feelings". Items from the CFQ-II included: "I get upset with myself for having certain thoughts" and "I spent a lot of time thinking about the past or future, rather than being engaged in activities that mattered to me." (Valuing Questionnaire, VQ; Smout, Davies, Burns, \& Christie, 2013). The initial items are shown in Table 1.

Items 1 to 13 are reverse-scored. Differing from the English version of the scale in which items were scored on a seven-point Likert-like scale, the items in this revised version were scored on a standard Likert scale (5 points), ranging from 1 ("strongly disagree") to 5 ("strongly agree"), with higher scores indicating better conditions.

Table 1

The initial items of the Ch-CompACT

\section{Behavioral awareness $\quad$ Valued action}

1. One of my big goals is $\quad 8$. It seems I am "running $\quad 14$. I make choices based to be free from painful on automatic" without on what is important to emotions. much awareness of what me, even if it is stressful. I'm doing.
2. I go out of my way to
9. Even when doing the
15. My values are really
avoid situations that
things that matter to me, I reflected in my behavior.
might bring difficult 
thoughts, feelings, or

sensations.

3. I worry about not being

able to control my worries

and feelings.

4. I work hard to keep out

upsetting feelings. find myself doing them

without paying attention.

10. I rush through

meaningful activities

without being really

attentive to them.

11. I do jobs or tasks

automatically, without

being aware of what I'm

doing.

5. My painful experiences

and memories make it

difficult for me to live a

life that I would value.

6. I get upset with myself

for having certain

thoughts.

7. I'm afraid of my

feelings.
13. I spent a lot of time

thinking about the past or

future, rather than being

engaged in activities that

mattered to me.
16. I am able to follow

my long terms plans

including times when

progress is slow.

17. When something is

important to me, I'll do it

even if there is a chance it

will upset me.

18. I behave in line with

my personal values.
19. I undertake things that

are meaningful to me,

even when I find it hard to

do so.

20. I act in ways that are

consistent with how I

wish to live my life. 


\section{Participants}

The study was designed as a cross-sectional survey. The participants were public security workers from all across the country. In cooperation with the Ministry of Public Security, we sent the scales through an online platform. Participants were invited to complete a self-report questionnaire on psychological flexibility, mental health, and life satisfaction on the internet. A total of 10,061 civil servants completed the questionnaire, while 7,842 of them were excluded from analysis due to missing values on the relevant items or lying on the questionnaire. The effective response rate was 56.19\%. We randomly divided the sample into two groups: Group 1 and Group 2. We used Group 1 in this study. Group 2 was used in another study to determine subgroups based on the psychological flexibility, source of pressure, and life satisfaction in Chinese civil servants (in prep). The purpose of the research was explained to the respondents before the research began and the questionnaire was anonymous.

\section{Instruments}

Psychological flexibility is related to decreased negative affect and enhanced well-being, therefore, in order to investigate the relationships between CompACT outcomes and negative emotions and well-being, all participants in our study completed the and the Depression, Anxiety, and Stress Scale (DASS-21; Lovibond \& Lovibond, 1996)) in addition to providing their basic demographic information. 
Depression Anxiety Stress Scales-21.The DASS-21 is used to evaluate an individual's level of negative emotion within the past week. The higher the score, the more serious the level of negative emotion. This is a self-report questionnaire that consists of 21 items, 7 items per subscale: Depression, Anxiety, and Stress. The Chinese version of the DASS-21 used in the present study has been validated. The Cronbach's alpha values were $0.83,0.80,0.82$, and 0.92 for the Depression, Anxiety, and Stress subscales, and the total scale, respectively (Wang et al., 2016). Unlike the original Chinese version of the DASS-21, in this study, participants were asked to score every item on a scale from 1 (did not apply to me at all) to 5 (applied to me very much).

Satisfaction With Life Scale. The SWLS is a five-item scale that assesses individual satisfaction with life. In the present study, we used the Chinese version translated by Xiong et al. The Cronbach's alpha was 0.78 (Xiong \& Xu, 2009). The SWLS is rated using a Likert-7 scoring system from 1 ("absolutely incorrect") to 7 ("totally correct"). The higher the score, the more satisfied the individual is with their life. We used a standard 1-5 Likert rating criteria in the present study.

\section{Statistical analyses}

We calculated Cronbach's $\alpha$ and conducted a Guttmann split-half test to assess the internal consistency and used Pearson's correlation to examine the correlations of all variables and items. In order to evaluate invariance of the scale and model-data fit, we randomly divided the Group 1 participants into two approximate halves and 
conducted exploratory factor analysis (EFA) on one and confirmatory factor analysis (CFA) on the other. We used SPSS 25.0 software (IBM Corp., Armonk, NY) to calculate the reliability, correlations, and CFA, and used SPSS AMOS Version 21 to conduct the CFA.

\section{Results}

\section{Participant characteristics}

Group1 consisted of 4, 999 civil servants. Their characteristics are shown in Table 1. Of the 4,999 respondents, $73.5 \%$ were male, and $26.5 \%$ were female. The average age was $36.6 \pm 8.26$ years. In terms of education level, the majority of the participants attended undergraduate programs (71.3\%). Additionally, most respondents were married (79.5\%). The total sample was randomly split into the EFA sample $(n=2,509)$ and the CFA sample $(n=2,490)$ by SPSS 25.0 during the factoranalysis process.

Table 2

Participant demographics

\begin{tabular}{lccc}
\hline Demographics & Total & EFA & CFA \\
sample & sample & sample \\
$(\mathrm{N}=$ & $(\mathrm{N}=$ & $(\mathrm{N}=$ \\
$4,999)$ & $2,509)$ & $2,490)$ \\
\hline
\end{tabular}




\begin{tabular}{|c|c|c|c|c|c|c|c|}
\hline & & $\mathrm{N}$ & $\%$ & $\mathrm{~N}$ & $\%$ & $\mathrm{~N}$ & $\%$ \\
\hline \multirow[t]{2}{*}{ Sex } & Female & 1,327 & $26.5 \%$ & 686 & $27.3 \%$ & 641 & $25.7 \%$ \\
\hline & Male & 3,672 & $73.5 \%$ & 1,823 & $72.7 \%$ & 1,849 & $74.3 \%$ \\
\hline \multirow[t]{7}{*}{ Age } & $(18.25)$ & 328 & $6.6 \%$ & 157 & $6.3 \%$ & 171 & $6.9 \%$ \\
\hline & $(25,30)$ & 1,068 & $21.4 \%$ & 562 & $22.4 \%$ & 506 & $20.3 \%$ \\
\hline & $(30,35)$ & 1,109 & $22.2 \%$ & 568 & $22.6 \%$ & 541 & $21.7 \%$ \\
\hline & $(35,40)$ & 902 & $18.0 \%$ & 440 & $17.5 \%$ & 462 & $18.6 \%$ \\
\hline & $(40,45)$ & 751 & $15.0 \%$ & 377 & $15.0 \%$ & 374 & $15.0 \%$ \\
\hline & $(45,50)$ & 534 & $10.7 \%$ & 248 & $9.9 \%$ & 286 & $11.5 \%$ \\
\hline & $(50,+\infty)$ & 307 & $6.1 \%$ & 157 & $6.3 \%$ & 150 & $6.0 \%$ \\
\hline \multirow{5}{*}{$\begin{array}{c}\text { Marital } \\
\text { status }\end{array}$} & Married & 3,974 & $79.5 \%$ & 1,988 & $79.2 \%$ & 1.986 & $79.8 \%$ \\
\hline & & & & & & & \\
\hline & Divorced & 209 & $4.2 \%$ & 111 & $4.4 \%$ & 98 & $3.9 \%$ \\
\hline & Widowed & 15 & $0.3 \%$ & 6 & $0.2 \%$ & 9 & $0.4 \%$ \\
\hline & Unmarried & 801 & $16.0 \%$ & 404 & $16.1 \%$ & 397 & $15.9 \%$ \\
\hline \multirow{5}{*}{$\begin{array}{c}\text { Education } \\
\text { level }\end{array}$} & $\mathrm{MD} / \mathrm{PhD}$ & 9 & $0.2 \%$ & 4 & $0.2 \%$ & 5 & $0.2 \%$ \\
\hline & & & & & & & \\
\hline & MA/MS & 241 & $4.8 \%$ & 117 & $4.7 \%$ & 124 & $5.0 \%$ \\
\hline & Undergraduate & 3,566 & $71.3 \%$ & 1,802 & $71.8 \%$ & 1,764 & $70.8 \%$ \\
\hline & Junior College & 1,183 & $23.7 \%$ & 586 & $23.4 \%$ & 597 & $24 \%$ \\
\hline
\end{tabular}




\section{Factor analysis}

Exploratory Factor Analysis. Cronbach's $\alpha$ was between 0.81 and 0.87 when each item was deleted, indicating that each item was necessary and of equal importance. In the factor analysis process, the total sample $(n=4,999)$ was randomly split into the EFA sample $(n=2,509)$ and the CFA sample $(n=2,490)$ by SPSS 25.0. Using the data from the EFA sample, the calculated EFA was used to identify possible latent variables of the Ch-CompACT. The items with factor loading less than .45 or communality less than .40 were deleted. Item 6 and Item 14 were deleted because communality was less than .40 . Thus, 18 items remained. Table 2 shows the results of the EFA using the principal axis extraction method with varimax rotation. The Kaiser-Meyer-Olkin Index $(\mathrm{KMO}=0.91)$ and Bartlett sphericity test $\left(\chi^{2}\right.$ $=16,092.237, \mathrm{df}=153, p<.001)$ show that the data could be used to conduct a factor analysis. We extracted three factors. Factor 1: openness to experience; Factor 2: behavioral awareness; Factor 3: valued action. The eigenvalue of each factor was greater than 1, and the load factor was greater than .45 for each item. Factor 1 included six items and explained $19.4 \%$ of the variance. Factor 2 included six items and explained $17.4 \%$ of the variance. Factor 3 included six items and explained $17.5 \%$ of the variance. The three factors together explained $54.3 \%$ of the total variance. Table 3 shows good commonalities and loadings on the main factor ranging from .527 (Item 8) to .803 (Item 11).

Table 3 
Items and factor loadings for the CompACT

\begin{tabular}{|c|c|c|c|c|}
\hline \multirow{2}{*}{$\begin{array}{c}\text { Source of item and } \\
\text { content }\end{array}$} & \multicolumn{3}{|c|}{ Factor loading } & \multirow[t]{2}{*}{ Communalities } \\
\hline & 1 & 2 & 3 & \\
\hline 1. One of my big goals & 699 & .157 & .109 & .525 \\
\hline \multicolumn{5}{|l|}{ is to be free from } \\
\hline \multicolumn{5}{|l|}{ painful emotions. } \\
\hline 2. I go out of my way to & .772 & .111 & .015 & .608 \\
\hline \multicolumn{5}{|l|}{ avoid situations that } \\
\hline \multicolumn{5}{|l|}{ might bring difficult } \\
\hline \multicolumn{5}{|l|}{ thoughts, feelings, or } \\
\hline \multicolumn{5}{|l|}{ sensations. } \\
\hline 3. I worry about not & .762 & .219 & .164 & .655 \\
\hline \multicolumn{5}{|l|}{ being able to control } \\
\hline \multicolumn{5}{|l|}{ my worries and } \\
\hline \multicolumn{5}{|l|}{ feelings. } \\
\hline 4. I work hard to keep & .703 & .152 & .004 & .518 \\
\hline \multicolumn{5}{|l|}{ out upsetting feelings. } \\
\hline 5. My painful & .700 & .227 & .135 & .561 \\
\hline \multicolumn{5}{|l|}{ experiences and } \\
\hline \multicolumn{5}{|l|}{ memories make it } \\
\hline difficult for me to live & & & & \\
\hline
\end{tabular}


a life that I would

value.

7. I'm afraid of my

.628

.213

.091

.448

feelings.

8. It seems I am

.348

.527

.119

.413

"running on

automatic" without

much awareness of

what I'm doing.

9. Even when doing the

.085

.784

.103

.632

things that matter to

me, I find myself doing

them without paying

attention.

10. I rush through

.115

.791

.100

.649

meaningful activities

without being really

attentive to them.

11. I do jobs or tasks

.198

.803

.164

.711

automatically, without

being aware of what

I'm doing. 
$\begin{array}{llllll}\text { 12. I find it difficult to } & .258 & \mathbf{. 6 2 4} & .117 & .470\end{array}$

stay focused on

what's happening in

the present .

13. I spend a lot of time .33

.543

.152

thinking about the past

or future, rather than

being engaged in

activities that matter to

me.

15. My values are

.069

.100

.650

.437

really reflected in my

behavior.

16. I am able to follow

.070

.120

.751

.584

my long terms plans

even when progress is

slow.

17. When something is $\quad-.038 \quad 051 \quad .051$

important to me, I'll

do it even if there is a

chance it will upset me. 
18. I behave in line

with my personal

values.

19. I undertake thing

that are meaningful to

me, even when I find it

hard to do so.

20. I act in ways that

\section{how I wish to live my}

life.

$\%$ of variance

Cumulative \% of
$19.4 \%$

$19.4 \%$

\section{are consistent with}

variance

\section{Confirmatory Factor Analysis (CFA). Having obtained the factor structure for} the EFA, we used the other half of Group $1(n=2,490)$ to perform a CFA of the factor model revealed by exploratory analysis. We performed maximum-likelihood confirmatory factor analyses using AMOS 21 to examine the proposed three-factor structure of the CompACT. The model contained the following fit indices (Table 5): the ratio of the minimum fit chi-square function to the degrees of freedom $\left(\chi^{2} / d f\right)$, the Bentler-Bonett normed fit index (NFI), the incremental fit index (IFI), the TuckerLewis index (TLI), the comparative fit index (CFI), the parsimony-adjusted 
comparative fit index (PCFI), and the root mean square error of approximation (RMSEA). According to previous studies, a good model fit is indicated by a $\chi^{2} / \mathrm{df}$ value smaller than 3.0, NFI, IFI, TLI, and CFI indices above 0.90 , and a PCFI above 0.50. For the RMSEA, values below 0.05 indicate a good fit, values between 0.05 and 0.08 indicate an acceptable fit, and values greater than 0.10 indicate a poor fit (Medsker, Williams, \& Holahan, 1994). The statistical results indicated that the models were fitted to the actual data: $\chi^{2} / d f=6.923, \mathrm{NFI}=.946, \mathrm{IFI}=.934$, TLI $=.943, \mathrm{CFI}=.954, \mathrm{PCFI}=.779, \mathrm{RMSEA}=.049$. This model is shown as Fig 1 . Table 4

Goodness-of-fit indices for the Ch-CompACT

\begin{tabular}{lllllll}
\hline$\chi^{2} / \boldsymbol{d} \boldsymbol{f}$ & NFI & IFI & TLI & CFI & PCFI & RMSEA \\
\hline $865.392 / 125=$ & .946 & .954 & .943 & .954 & .779 & .049 \\
6.923 & & & & & & \\
\hline
\end{tabular}




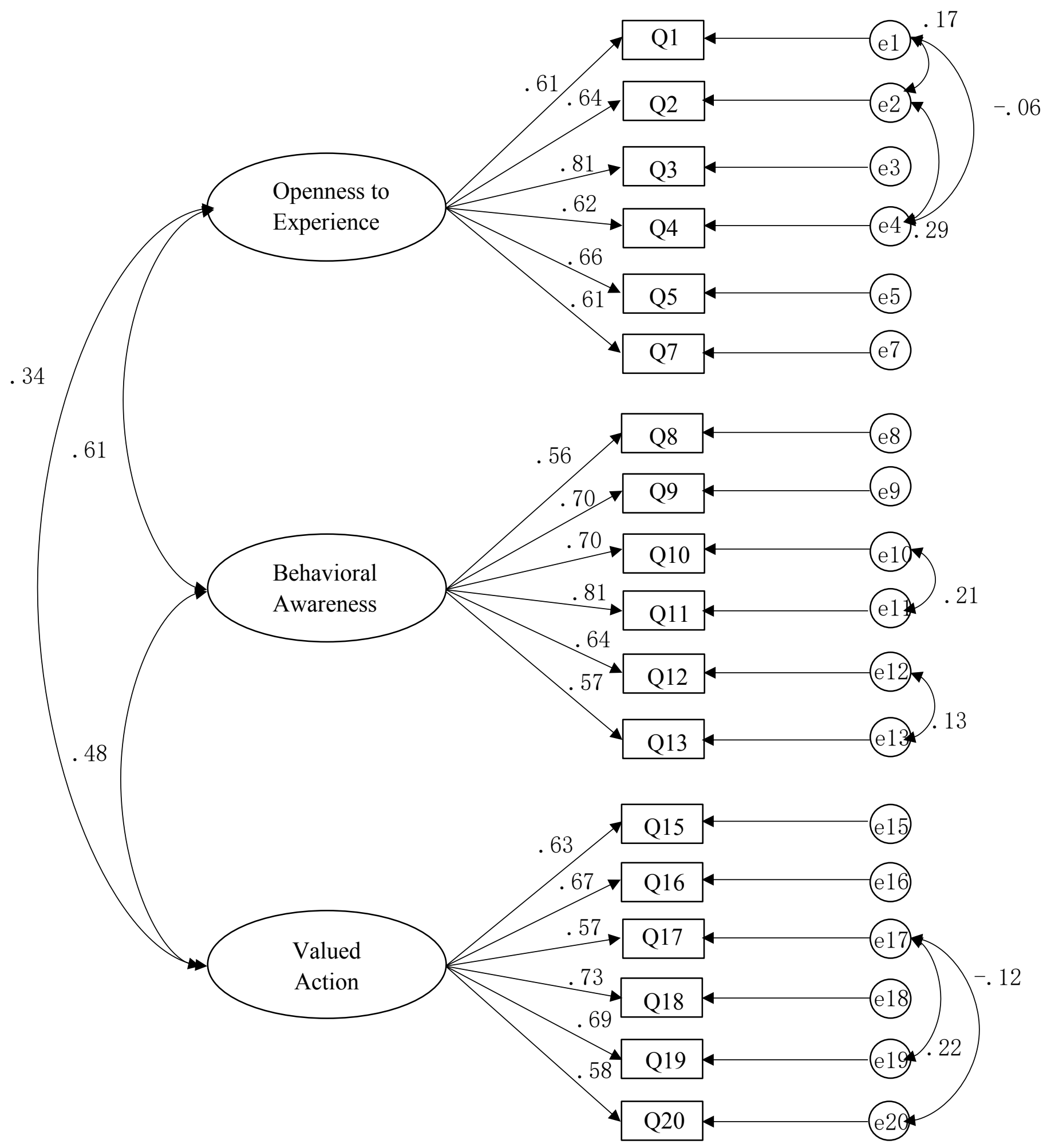

Fig.1 .Measurement model for Ch-CompACT 
Q1

Consistency and Reliability. Cronbach's alpha for the Ch-CompACT was 0.87 $(>0.8)$, which was obtained using the Spearman-Brown formula. This value indicates that the scale has a good reliability. We then calculated Cronbach's $\alpha$ for each subscale of the Ch-CompACT, with acceptable results (Table 5). We then conducted Guttmann split-half tests, which indicated reliabilities ranging from 0.69 to 0.84 for the total scale and for the individual subscales.

Table 5

Cronbach's $\alpha$ and the Guttmann split-half coefficient for each subscale of the Ch-

CompACT and for the Ch-CompACT as a whole

\begin{tabular}{lcc}
\hline & Cronbach's $\alpha$ & Guttman split- \\
& .83 & .84 \\
\hline half coefficient \\
Experience & .83 & \\
Behavioral & & \\
Awareness & .84 \\
Valued Action & .81 & .79 \\
CompACT Total & .87 & \\
Score & \\
\hline
\end{tabular}

Correlations Between the Ch-CompACT and other Measures 
We evaluated the Pearson's correlation coefficients between the scores for each subscale of the Ch-CompACT and those on the DASS, SWLS, and SCL-90 in order to explore the relationships between Ch-CompACT scores and psychological symptoms and well-being variables. Table 6 shows that all factors of the ChCompACT correlated significantly with the SWLS (positively) and with the DASS (negatively).

Table 6

Correlations Between the CompACT and Other Variables

\begin{tabular}{lcccc}
\hline Measure & \multicolumn{2}{c}{ Correlation (r) } & & \\
\cline { 2 - 5 } & CompACT & CompACT & CompACT & CompACT \\
& Total Score & Openness to & Behavioral & Valued Action \\
\hline SWLS & & experience & Awareness & \\
DASS-21 & $.55^{* *}$ & $.44^{* *}$ & $.35^{* *}$ & $.48^{* *}$ \\
Depression & $-.71^{* *}$ & $-.61^{* *}$ & & $-.44^{* *}$ \\
Anxiety & $-.64^{* *}$ & $-.61^{* *}$ & $-.58^{* *}$ & $-.33^{* *}$ \\
Stress & $-.66^{* *}$ & $-.66^{* *}$ & $-.51^{* *}$ & $-.31^{* *}$ \\
\hline
\end{tabular}

Note. SWLS, Satisfaction With Life Scale; DASS-21, Depression Anxiety and Stress Scales, 21-item version

$* * p<.01$ 


\section{Discussion}

Recent intervention studies have emphasized the importance of examining the mechanisms underlying the changes in outcomes $(\mathrm{Gu}$, Strauss, Bond, \& Cavanagh, 2015). Past empirical studies have suggested that psychological flexibility mediates changes in overall well-being and psychological symptoms (Puolakanaho, Tolvanen, Kinnunen, \& Lappalainen, 2020; Bardeen, Fergus, \& Orcutt, 2013). However, reliable and valid instruments are needed to comprehensively explore psychological flexibility. The current study analyzed the reliability and validity of the Chinese version of the CompACT using a sample of Chinese civil servants working in the public security system. Even though several items and scoring criteria in the ChCompACT differ from the original, the results indicate that the Ch-CompACT has a relatively reasonable 18-item, 3-factor structure of psychological flexibility, which is comparable to the English version (Francis et al., 2016).

In terms of reliability, Cronbach's alpha for the Ch-CompACT was 0.87 , and the split-half coefficients was 0.69 . The alpha values for the subscales ranged from 0.81 to 0.83 , and split-half coefficients ranged from 0.79 to 0.84 . These results indicated satisfactory reliability and internal consistency of the Ch-CompACT. The EFA extracted three factors from the Ch-CompACT, which could collectively explain $54.3 \%$ of the overall variance. Factor loadings ranged from .527 to .803 , and each factor contained 6 items. 
Unlike the original version of the CompACT, which contains no items originating from the AAQ-II, we used three items from the AAQ-II in the ChCompACT. During the initial revision of the CompACT, we removed some items because we felt they would be difficult to understand in the Chinese cultural context. Therefore, it was necessary to add several items to expand the Openness to Experience subscale. The AAQ-II did not load on other domains of psychological flexibility except for acceptance/experiential avoidance and diffusion/fusion processes. The Chinese version of the AAQ-II was translated by Cao et al.(2013), and has shown good reliability and validity (Cao, Ji, \& Zhu, 2013). Considering that the purpose of the CompACT is to comprehensively measure psychological flexibility, and that the AAQ-II has been proved useful as a tool to measure individual experiential avoidance, we accepted the expert suggestion and selected 3 items from the Chinese version of AAQ-II that reflect the individual's acceptance of their experience, and added them to the Openness to Experience subscale. The results show that these items are consistent with the subscale and the total score of the scale.

In ACT interventions, positive changes are thought to be derived from changes in processes related to psychological flexibility (Hayes et al., 2006; Hayes et al., 2012). At the same time, psychological flexibility has been shown to be an important determinant of mental health (Bai et al., 2019; Coto-Lesmes, Fernández-Rodríguez, \& González-Fernández, 2020). Thus, studying the detailed relationship between psychological flexibility and mental health is important. The results of this study were consistent with former researches. The three subscales and the total score were 
correlated to the SWLS, and as in the English version, were negatively correlated with the DASS-21. Individuals with higher levels of psychological flexibility are more able to be aware of their present activities, and are more nonjudgmental of their emotions, feelings, and thoughts. Thus, they can take more effective actions and are less likely to experience negative emotions and complain about their lives. These results implied that scores on the Ch-CompACT can predict how much psychological flexibility facilitates emotional regulation and well-being outcomes.

This is the first study of CompACT factor structure on Chinese civil servants. Participants came from provinces and cities with different levels of economic development across the country. A large number of studies have shown that civil servants in various regions of China have different levels of anxiety, depression, burnout, and other psychological problems due to particular aspects of their work and environment (Li et al., 2013; Xu, Zhao, Wang, \& Sun, 2010; Hao et al., 2014; Hao, Hong, Xu, Zhou, \& Xie, 2015b). Ethnicity, resilience, and demographic characteristics are all considered to be factors affecting the mental health of civil servants (Fu et al., 2017; Hao et al., 2015b; Hong, Xie, Zhou, Hao, \& Xu, 2015; Huang et al., 2018). Poor psychological flexibility is an important cause of many psychological and behavioral problems (Zhang, Wang, \& Zhu, 2012). Research has been lacking on the psychological flexibility of civil servants and its relationship with demographic characteristics. This Chinese revised version of the scale will provide an effective tool for this type of research. 
This study has some limitations. First, participants were all civil servants, who face special professional circumstances and work environments. Therefore, the results may not be applicable to the general population or other occupational groups. Furthermore, more studies need to be conducted to confirm the scale's psychometric properties and performance among a more diverse population. Additionally, participants in this study came from all over the country, which made the results consistent with the characteristics of Chinese context. However, the large sample size and the regional diversity may result in poor fitting index results $\left(\chi^{2} / d f>3\right)$. Future studies can verify this questionnaire in populations with smaller sample sizes and more uniform regions.

\section{Conclusions}

In summary, the current study found that the Ch-CompACT has acceptable psychometric properties. It is a valid instrument for evaluating different aspects of psychological flexibility. 


\section{Acknowledgements}

This work was supported by the China Postdoctoral Science Foundation [grant numbers 2016M601156]

\section{References}

Bai, Z., Luo, S., Zhang, L., Wu, S., \& Chi, I. (2019). Acceptance and Commitment Therapy (ACT) to reduce depression: A systematic review and meta-analysis. Journal of Affective Disorders. https://doi.org/10.1016/j.jad.2019.09.040

Bardeen, J. R., Fergus, T. A., \& Orcutt, H. K. (2013). Experiential Avoidance as a Moderator of the Relationship Between Anxiety Sensitivity and Perceived Stress. Behavior Therapy, 44(3), 459-469. https://doi.org/10.1016/j.beth.2013.04.001

Bond, F. W., Hayes, S. C., Baer, R. A., Carpenter, K. M., Guenole, N., Orcutt, H. K., ... Zettle, R. D. (2011). Preliminary Psychometric Properties of the Acceptance and Action Questionnaire-II: A Revised Measure of Psychological Inflexibility and Experiential Avoidance. Behavior Therapy, 42(4), 676-688. https://doi.org/10.1016/j.beth.2011.03.007

Cao, J., Ji, Y., \& Zhu, Z. (2013). Reliability and validity of the Chinese version of the Acceptance and Action Questionnaire-Second Edition (AAQ-II) in college students. Chinese Mental Health Journal, 27(11), 3-7. 
Chen, C. (2005). Mental health of the civil servants. Development Research, 19(4), 71-73. https://doi.org/10.1016/j.hbrcj.2014.06.002

Coto-Lesmes, R., Fernández-Rodríguez, C., \& González-Fernández, S. (2020). Acceptance and Commitment Therapy in group format for anxiety and depression. A systematic review. Journal of Affective Disorders, 263, 107-120. https://doi.org/10.1016/j.jad.2019.11.154

Dindo, L., Van Liew, J. R., \& Arch, J. J. (2017). Acceptance and Commitment Therapy: A Transdiagnostic Behavioral Intervention for Mental Health and Medical Conditions. Neurotherapeutics, 14(3), 546-553. https://doi.org/10.1007/s13311-017-0521-3

Francis, A. W., Dawson, D. L., \& Golijani-Moghaddam, N. (2016). The development and validation of the Comprehensive assessment of Acceptance and Commitment Therapy processes (CompACT). Journal of Contextual Behavioral Science, 5(3), 134-145. https://doi.org/10.1016/j.jcbs.2016.05.003

Fu, A., Liu, B., Jiang, Y., Zhao, J., Zhang, G., \& Liu, J. (2017). A mental health survey of different ethnic and occupational groups in Xinjiang, China. International Journal of Environmental Research and Public Health, 14(1). https://doi.org/10.3390/ijerph14010046

Gámez, W., Chmielewski, M., Kotov, R., Ruggero, C., Suzuki, N., \& Watson, D. (2014). The brief experiential avoidance questionnaire: Development and initial 
validation. Psychological Assessment, 26(1), 35-45.

https://doi.org/10.1037/a0034473

Gillanders, D. T., Bolderston, H., Bond, F. W., Dempster, M., Flaxman, P. E., Campbell, L., ... Remington, B. (2014). The Development and Initial Validation of the Cognitive Fusion Questionnaire. Behavior Therapy, 45(1), 83-101. https://doi.org/10.1016/j.beth.2013.09.001

Gu, J., Strauss, C., Bond, R., \& Cavanagh, K. (2015). How do mindfulness-based cognitive therapy and mindfulness-based stress reduction improve mental health and wellbeing? A systematic review and meta-analysis of mediation studies. Clinical Psychology Review, 37, 1-12. https://doi.org/10.1016/j.cpr.2015.01.006

Hao, S., Hong, W., Xu, H., Zhou, L., \& Xie, Z. (2015a). Relationship between resilience, stress and burnout among civil servants in Beijing, China: Mediating and moderating effect analysis. Personality and Individual Differences, 83, 6571. https://doi.org/10.1016/j.paid.2015.03.048

Hao, S., Hong, W., Xu, H., Zhou, L., \& Xie, Z. (2015b). Relationship between resilience, stress and burnout among civil servants in Beijing, China: Mediating and moderating effect analysis. Personality and Individual Differences, 83, 6571. https://doi.org/10.1016/j.paid.2015.03.048

Hao, S., Zhou, L., Sun, S., Xu, H., Xie, Z., \& Hong, W. (2014). Mental Health,Job Burnout of Civil Servants and Their Influencing Factors. Journal of Chemical 
Information and Modeling, 22(6), 844-846.

https://doi.org/10.1017/CBO9781107415324.004

Hayes, S. C. (2004). Acceptance and Commitment Therapy, Relational Frame

Theory, and the Third Wave of Behavioral and Cognitive Therapies -

Republished Article. Behavior Therapy, 35, 639-665.

https://doi.org/10.1016/j.beth.2016.11.006

Hayes, S. C., \& Hofmann, S. G. (2017). The third wave of cognitive behavioral therapy and the rise of process-based care. World Psychiatry, 16(3), 245-246. https://doi.org/10.1002/wps.20442

Hayes, S. C., Levin, M. E., Plumb-Vilardaga, J., Villatte, J. L., \& Pistorello, J. (2013). Acceptance and commitment therapy and contextual behavioral science: Examining the progress of a distinctive model of behavioral and cognitive therapy. Behavior Therapy, 44(2), 180-198.

https://doi.org/10.1161/CIRCULATIONAHA.110.956839

Hayes, S. C., Luoma, J. B., Bond, F. W., Masuda, A., \& Lillis, J. (2006). Acceptance and Commitment Therapy : Model, processes and outcomes. Behaviour Research and Therapy, 44(1), 1-25. https://doi.org/10.1016/j.brat.2005.06.006

Hayes, S. C., Pistorello, J., \& Levin, M. E. (2012). Acceptance and Commitment Therapy as a Unified Model of Behavior Change. The Counseling Psychologist, 40(7), 976-1002. https://doi.org/10.1177/0011000012460836 
Hayes, S. C., Villatte, M., Levin, M., \& Hildebrandt, M. (2011). Open, Aware, and Active: Contextual Approaches as an Emerging Trend in the Behavioral and Cognitive Therapies. Annual Review of Clinical Psychology, 7(4), 141-168. https://doi.org/10.1146/annurev-clinpsy-032210-104449

Hofmann, S. G., \& Hayes, S. C. (2019). The Future of Intervention Science: ProcessBased Therapy. Clinical Psychological Science, 7(1), 37-50. https://doi.org/10.1177/2167702618772296

Hong, W., Xie, Z., Zhou, L., Hao, S., \& Xu, H. (2015). Effect of Resilience on Depression and Anxiety Symptoms in Chinese Grassroots Civil Servants. Chinese Journal of Clinical Psychology, 23(5), 795-798. https://doi.org/10.16128/j.cnki.1005-3611.2015.05.008

Huang, X., Cao, Q., Liu, L., Yao, J., \& Rui, G. (2018). Factors Influencing Depression in Chinese Civil Servants. Social Behavior and Personality, 46(8), $1233-1243$.

Jia, Y., Gao, J., Dai, J., Zheng, P., Wu, X., Li, G., \& Fu, H. (2014). Difference of the associations between self-rated health and demographic characteristics, lifestyle, and psychosocial work environment between two types of Chinese worksite. BMC Public Health, 14(851), 1-12. https://doi.org/10.1186/1471-2458-14-851

Lee, E. B., Homan, K. J., Morrison, K. L., Ong, C. W., Levin, M. E., \& Twohig, M. P. (2018). Acceptance and Commitment Therapy for Trichotillomania: A 
Randomized Controlled Trial of Adults and Adolescents. Behavior Modification, 44(1), 70-91. https://doi.org/10.1177/0145445518794366

Li, J., Lv, L., Zhang, Z., Guo, Z., Wang, K., Wang, D., ... Tao, M. (2013).

Psychological and physiological health and social support among different populations in urban areas of Ningxia. Chinese Journal of Public Health, 29(03), $412-414$.

Li, Y. (2014). Analysis of Reasons for Chinese Civil Servants Resigning from Office. In PROCEEDINGS OF THE 2014 INTERNATIONAL CONFERENCE ON PUBLIC MANAGEMENT (Vol. 650221, pp. 265-274). https://doi.org/10.2991/icpm-14.2014.48

Liang, Y., Wang, L., \& Yin, X. (2016). The factor structure of the 12-item general health questionnaire (GHQ-12) in young Chinese civil servants. Health and Quality of Life Outcomes, 14(1), 1-9. https://doi.org/10.1186/s12955-016-0539-y

Liu, Y., \& Liu, Y. (2006). Current Researches on Civil Servants' Mental Health. JOURNAL OF LINYI TEACHERS' UNIVERSITY, 28(4), 83-86.

Livheim, F., Hayes, L., Ghaderi, A., Magnusdottir, T., Högfeldt, A., Rowse, J., ... Tengström, A. (2015). The Effectiveness of Acceptance and Commitment Therapy for Adolescent Mental Health: Swedish and Australian Pilot Outcomes. Journal of Child and Family Studies, 24(4), 1016-1030. https://doi.org/10.1007/s10826-014-9912-9 
Lovibond, P. F., \& Lovibond, S. H. (1996). The structure of negative emotional states: Comparison of the Depression Anxiety Stress Scales (DASS) with the Beck Depression and Anxiety Inventories. Medical and Biological Engineering and Computing, 34(4), 313-315. https://doi.org/10.1007/BF02511245

Lu, P., \& Liang, Y. (2016). Health-Related Quality of Life of Young Chinese Civil Servants Working in Local Government: Comparison of SF-12 and EQ5D. Applied Research in Quality of Life, 11(4), 1445-1464. https://doi.org/10.1007/s11482-015-9446-3

McLean, C., \& Follette, V. M. (2016). Acceptance and commitment therapy as a nonpathologizing intervention approach for survivors of trauma. Journal of Trauma and Dissociation, 17(2), 138-150.

https://doi.org/10.1080/15299732.2016.1103111

Medsker, G. J., Williams, L. J., \& Holahan, P. J. (1994). A Review of Current Practices for Evaluating Causal Models in Organizational Behavior and Human Resources Management Research. Journal of Management, 20(2), 439-464. https://doi.org/10.1177/014920639402000207

Nian, C. (2004). Standards and countermeasures of civil servants' mental health. Science of Social Psychology, 4, 95-98.

Puolakanaho, A., Tolvanen, A., Kinnunen, S. M., \& Lappalainen, R. (2020). A psychological flexibility -based intervention for Burnout:A randomized 
controlled trial. Journal of Contextual Behavioral Science, (15), 52-67.

https://doi.org/10.1016/j.jcbs.2019.11.007

Smout, M., Davies, M., Burns, N., \& Christie, A. (2013). Development of the Valuing Questionnaire (VQ). Journal of Contextual Behavioral Science, 3(3), 164-172. https://doi.org/10.1016/j.jcbs.2014.06.001

Wang, K., Shi, H. S., Geng, F. L., Zou, L. Q., Tan, S. P., Wang, Y., ... Chan, R. C. K. (2016). Research on translations of tests: Cross-cultural validation of the depression anxiety stress scale-21 in China. Psychological Assessment, 28(5), e88-e100. https://doi.org/10.1037/pas0000207

Wang, Y. (2008). The influential factors and intervention mechanism of public servant's occupational health psychology. Chinese Public Administration, 9, 6164.

Wilson, K. G., Sandoz, E. K., Kitchens, J., \& Roberts, M. (2010). The valued living questionnaire: Defining and measuring valued action within a behavioral framework. Psychological Record, 60(2), 249-272. https://doi.org/10.1007/BF03395706

Wolgast, M. (2014). What does the acceptance and action questionnaire (AAQ-II) really measure? Behavior Therapy, 45(6), 831-839. https://doi.org/10.1016/j.beth.2014.07.002 
Xiong, C., \& Xu, Y. (2009). Reliability and Validity of the Stisfaction with Life Scale for Chinese Demos. China Journal of Health Psychology, 17(08), 948-949. https://doi.org/10.1017/CBO9781107415324.004

Xu, H., Zhao, H., Wang, S., \& Sun, Z. (2010). Association between Mental Health and Coping style of Public Functionaries in Beijing. China Journal of Health Psychology, 18(4), 426-428. https://doi.org/10.1017/CBO9781107415324.004

Zhang, Q., Wang, S., \& Zhu, Z. (2012). Acceptance and commitment therapy ( ACT) : Psychopathological model and processes of change. Chinese Mental Health Journal, 26(05), 377-381.

Zhu, C., Chen, L., Ou, L., Geng, Q., \& Jiang, W. (2014). Relationships of Mental Health Problems with Stress Among Civil Servants in Guangzhou, China. Community Mental Health Journal, 50(8), 991-996. https://doi.org/10.1007/s10597-014-9726-7 


\title{
Appendices
}

\author{
A.Tables
}

Table A.1

The initial items of the Ch-CompACT

\section{Behavioral awareness $\quad$ Valued action}

1. One of my big goals is

8. It seems I am "running

14. I make choices based

to be free from painful

on automatic" without

on what is important to

emotions.

much awareness of what

me, even if it is stressful.

I'm doing.

2. I go out of my way to

9. Even when doing the

15. My values are really

avoid situations that

things that matter to me, I reflected in my behavior.

might bring difficult

find myself doing them

thoughts, feelings, or

without paying attention.

sensations.

3. I worry about not being 10 . I rush through

16. I am able to follow

able to control my worries

meaningful activities

my long terms plans

and feelings.

without being really

including times when

attentive to them.

progress is slow.

4. I work hard to keep out

11. I do jobs or tasks

17. When something is

upsetting feelings.

automatically, without

important to me, I'll do it 
being aware of what I'm even if there is a chance it

doing. will upset me.

5. My painful experiences 12. I find it difficult to 18. I behave in line with

and memories make it stay focused on what's my personal values.

difficult for me to live a happening in the present.

life that I would value.

6. I get upset with myself 13 . I spent a lot of time 19. I undertake things that

for having certain thinking about the past or are meaningful to me,

thoughts. future, rather than being even when I find it hard to

engaged in activities that do so.

mattered to me.

7. I'm afraid of my

feelings.
20. I act in ways that are

consistent with how I

wish to live my life.

Table A. 2

Participant demographics

Demographics
Total

sample
EFA

CFA

sample sample 


\begin{tabular}{|c|c|c|c|c|c|c|c|}
\hline & & $(N=$ & & $(\mathbf{N}=$ & & $(\mathbf{N}=$ & \\
\hline & & 4,999) & & $2,509)$ & & $2,490)$ & \\
\hline & & $\mathrm{N}$ & $\%$ & $\mathrm{~N}$ & $\%$ & $\mathrm{~N}$ & $\%$ \\
\hline \multirow[t]{2}{*}{ Sex } & Female & 1,327 & $26.5 \%$ & 686 & $27.3 \%$ & 641 & $25.7 \%$ \\
\hline & Male & 3,672 & $73.5 \%$ & 1,823 & $72.7 \%$ & 1,849 & $74.3 \%$ \\
\hline \multirow[t]{7}{*}{ Age } & $(18.25)$ & 328 & $6.6 \%$ & 157 & $6.3 \%$ & 171 & $6.9 \%$ \\
\hline & $(25,30)$ & 1,068 & $21.4 \%$ & 562 & $22.4 \%$ & 506 & $20.3 \%$ \\
\hline & $(30,35)$ & 1,109 & $22.2 \%$ & 568 & $22.6 \%$ & 541 & $21.7 \%$ \\
\hline & $(35,40)$ & 902 & $18.0 \%$ & 440 & $17.5 \%$ & 462 & $18.6 \%$ \\
\hline & $(40,45)$ & 751 & $15.0 \%$ & 377 & $15.0 \%$ & 374 & $15.0 \%$ \\
\hline & $(45,50)$ & 534 & $10.7 \%$ & 248 & $9.9 \%$ & 286 & $11.5 \%$ \\
\hline & $(50,+\infty)$ & 307 & $6.1 \%$ & 157 & $6.3 \%$ & 150 & $6.0 \%$ \\
\hline \multirow{5}{*}{$\begin{array}{l}\text { Marital } \\
\text { status }\end{array}$} & Married & 3,974 & $79.5 \%$ & 1,988 & $79.2 \%$ & 1.986 & $79.8 \%$ \\
\hline & & & & & & & \\
\hline & Divorced & 209 & $4.2 \%$ & 111 & $4.4 \%$ & 98 & $3.9 \%$ \\
\hline & Widowed & 15 & $0.3 \%$ & 6 & $0.2 \%$ & 9 & $0.4 \%$ \\
\hline & Unmarried & 801 & $16.0 \%$ & 404 & $16.1 \%$ & 397 & $15.9 \%$ \\
\hline \multirow{5}{*}{$\begin{array}{c}\text { Education } \\
\text { level }\end{array}$} & $\mathrm{MD} / \mathrm{PhD}$ & 9 & $0.2 \%$ & 4 & $0.2 \%$ & 5 & $0.2 \%$ \\
\hline & & & & & & & \\
\hline & MA/MS & 241 & $4.8 \%$ & 117 & $4.7 \%$ & 124 & $5.0 \%$ \\
\hline & Undergraduate & 3,566 & $71.3 \%$ & 1,802 & $71.8 \%$ & 1,764 & $70.8 \%$ \\
\hline & Junior College & 1,183 & $23.7 \%$ & 586 & $23.4 \%$ & 597 & $24 \%$ \\
\hline
\end{tabular}


Table A.3

Items and factor loadings for the CompACT

\begin{tabular}{|c|c|c|c|c|}
\hline \multirow{2}{*}{$\begin{array}{c}\text { Source of item and } \\
\text { content }\end{array}$} & \multicolumn{3}{|c|}{ Factor loading } & \multirow[t]{2}{*}{ Communalities } \\
\hline & 1 & 2 & 3 & \\
\hline 1. One of my big goals & .699 & .157 & .109 & .525 \\
\hline \multicolumn{5}{|l|}{ is to be free from } \\
\hline \multicolumn{5}{|l|}{ painful emotions. } \\
\hline 2. I go out of my way to & .772 & .111 & .015 & .608 \\
\hline \multicolumn{5}{|l|}{ avoid situations that } \\
\hline \multicolumn{5}{|l|}{ might bring difficult } \\
\hline \multicolumn{5}{|l|}{ thoughts, feelings, or } \\
\hline \multicolumn{5}{|l|}{ sensations. } \\
\hline 3. I worry about not & .762 & .219 & .164 & .655 \\
\hline \multicolumn{5}{|l|}{ being able to control } \\
\hline \multicolumn{5}{|l|}{ my worries and } \\
\hline \multicolumn{5}{|l|}{ feelings. } \\
\hline 4. I work hard to keep & .703 & .152 & .004 & .518 \\
\hline
\end{tabular}


5. My painful $\quad \mathbf{7 0}$

$.700 \quad .22$

.135

.561

experiences and

memories make it

difficult for me to live

a life that I would

value.

7. I'm afraid of my

.628

.213

.091

.448

feelings.

8. It seems I am

.348

.527

.119

.413

"running on

automatic" without

much awareness of

what I'm doing.

9. Even when doing the

.085

.784

.103

.632

things that matter to

me, I find myself doing

them without paying

attention.

10. I rush through

.115

.791

.100

.649

meaningful activities

without being really

attentive to them. 


$\begin{array}{llllll}\text { 11. I do jobs or tasks } & .198 & \mathbf{8 0 3} & .164 & .711\end{array}$

automatically, without

being aware of what

I'm doing.

12. I find it difficult to .258

.624

.117

.470

stay focused on

what's happening in

the present .

13. I spend a lot of time .33

.543

.152

thinking about the past

or future, rather than

being engaged in

activities that matter to

me.

15. My values are

.069

.100

.650

.437

really reflected in $\mathrm{my}$

behavior.

16. I am able to follow

.070

.120

.751

.584

my long terms plans

even when progress is

slow. 
17. When something is $\quad-.038 \quad .05$

.721

.524

important to me, I'll

do it even if there is a

chance it will upset me.

18. I behave in line

.748

.600

with my personal

values.

19. I undertake things $\quad .034 \quad .140$

.759

that are meaningful to

me, even when I find it

hard to do so.

20. I act in ways that

.255

.111

.581

.415

are consistent with

how I wish to live my

life.

$\%$ of variance

$19.4 \%$

$17.4 \%$

$17.5 \%$

Cumulative $\%$ of

$19.4 \% \quad 36.8 \%$

$54.3 \%$

variance

Table A.4

Goodness-of-fit indices for the Ch-CompACT 


\begin{tabular}{lllllll}
\hline$\chi^{2} / \boldsymbol{d} \boldsymbol{f}$ & NFI & IFI & TLI & CFI & PCFI & RMSEA \\
\hline $865.392 / 125=$ & .946 & .934 & .943 & .954 & .779 & .049 \\
6.923 & & & & & & \\
\hline
\end{tabular}

Note: $\chi^{2} /$ :chi-square discrepancy, $d f:$ degrees of freedom, NFI: Normed Fit Index;

IFI: Incremental Fit Index,TLI: Tucker-Lewis index, CFI: Comparative Fit Index , PCFI: Parsimony-adjusted Comparative Fit Index, RMSEA: Root Mean Square Error of Approximation.

Table A.5

Cronbach's $\alpha$ and the Guttmann split-half coefficient for each subscale of the Ch-

CompACT and for the Ch-CompACT as a whole

\begin{tabular}{lcc}
\hline & Cronbach's $\alpha$ & Guttman split- \\
& .83 & .84 \\
\hline Openness to & & \\
Experience & .83 & .84 \\
Behavioral & & \\
Awareness & .79 \\
Valued Action & .81 & .69 \\
CompACT Total & .87 & \\
Score & \\
\hline
\end{tabular}


Table A.6

Correlations Between the CompACT and Other Variables

\begin{tabular}{lcccc}
\hline Measure & \multicolumn{2}{c}{ Correlation $(\mathbf{r})$} & & \\
\cline { 2 - 5 } & CompACT & CompACT & CompACT & CompACT \\
& Total Score & Openness to & Behavioral & Valued Action \\
& & experience & Awareness & \\
SWLS & $.55^{* *}$ & $.44^{* *}$ & $.35^{* *}$ & $.48^{* *}$ \\
DASS-21 & & & & $-.44^{* *}$ \\
Depression & $-.71^{* *}$ & $-.61^{* *}$ & $-.58^{* *}$ & $-.33^{* *}$ \\
Anxiety & $-.64^{* *}$ & $-.61^{* *}$ & $-.51^{* *}$ & $-.31^{* *}$ \\
\hline Stress & $-.66^{* *}$ & $-.66^{* *}$ & $-.51^{* *}$ & \\
\hline
\end{tabular}

Note. the Satisfaction With Life Scale(SWLS) is from Xiong et al.(2009); DASS-21, the Depression Anxiety and Stress Scales 21-item version (DASS-21) is from Wang et al.(2016).

$* * p<.01$ 


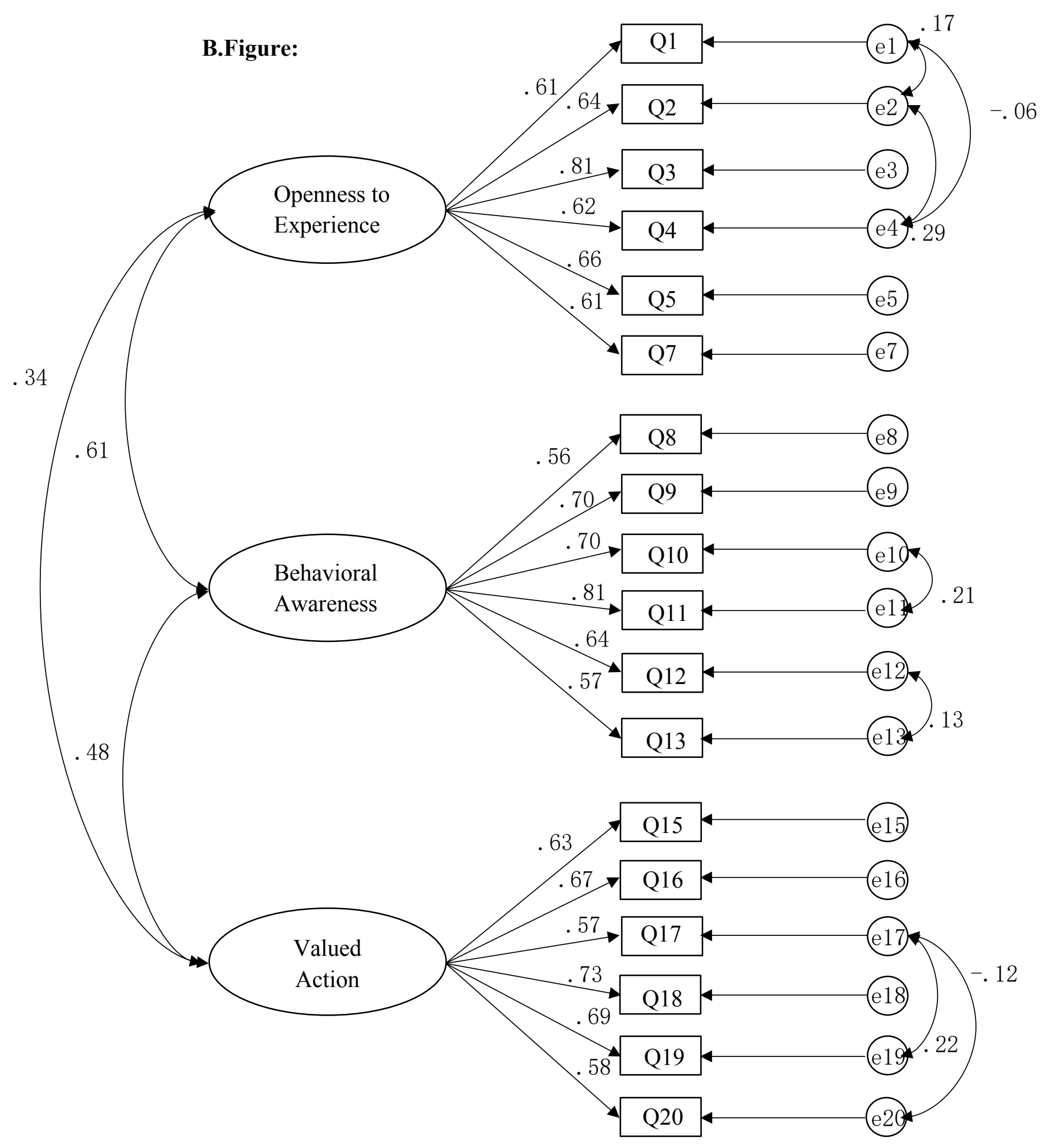

Fig.B.1 .Measurement model for Ch-CompACT 




Figure 1

Measurement model for Ch-CompACT 
This is a list of supplementary files associated with this preprint. Click to download.

- Tables.pdf 